\title{
Self-assembling influenza nanoparticle vaccines drive extended germinal center activity and memory $B$ cell maturation
}

\author{
Hannah G. Kelly, ${ }^{1,2}$ Hyon-Xhi Tan, ${ }^{1}$ Jennifer A. Juno, ${ }^{1}$ Robyn Esterbauer, ${ }^{1,2}$ Yi Ju, ${ }^{2,3}$ Wenbo Jiang, \\ Verena C. Wimmer, ${ }^{4}$ Brigette C. Duckworth, ${ }^{5}$ Joanna R. Groom, ${ }^{5}$ Frank Caruso, ${ }^{2,3}$ Masaru Kanekiyo, ${ }^{6}$ \\ Stephen J. Kent, ${ }^{1,2,7}$ and Adam K. Wheatley ${ }^{1,2}$ \\ 'Department of Microbiology and Immunology, University of Melbourne, The Peter Doherty Institute for Infection and \\ Immunity, Melbourne, Victoria, Australia. ${ }^{2}$ ARC Centre of Excellence in Convergent Bio-Nano Science and Technology \\ and ${ }^{3}$ Department of Chemical Engineering, University of Melbourne, Parkville, Victoria, Australia. ${ }^{4}$ Centre for Dynamic \\ Imaging and ${ }^{5}$ Division of Immunology, Walter and Eliza Hall Institute of Medical Research, Department of Medical \\ Biology, University of Melbourne, Parkville, Victoria, Australia. ${ }^{6}$ Vaccine Research Center, National Institute of Allergy \\ and Infectious Diseases (NIAID), NIH, Bethesda, Maryland, USA. ${ }^{7}$ Melbourne Sexual Health Centre and Department of \\ Infectious Diseases, Alfred Hospital and Central Clinical School, Monash University, Melbourne, Victoria, Australia.
}

Protein-based, self-assembling nanoparticles elicit superior immunity compared with soluble protein vaccines, but the immune mechanisms underpinning this effect remain poorly defined. Here, we investigated the immunogenicity of a prototypic ferritin-based nanoparticle displaying influenza hemagglutinin (HA) in mice and macaques. Vaccination of mice with HA-ferritin nanoparticles elicited higher serum antibody titers and greater protection against experimental influenza challenge compared with soluble HA protein. Germinal centers in the draining lymph nodes were expanded and persistent following HA-ferritin vaccination, with greater deposition of antigen that colocalized with follicular dendritic cells. Our findings suggest that a highly ordered and repetitive antigen array may directly drive germinal centers through a B cell-intrinsic mechanism that does not rely on ferritin-specific $T$ follicular helper cells. In contrast to mice, enhanced immunogenicity of HA-ferritin was not observed in pigtail macaques, where antibody titers and lymph node immunity were comparable to soluble vaccination. An improved understanding of factors that drive nanoparticle vaccine immunogenicity in small and large animal models will facilitate the clinical development of nanoparticle vaccines for broad and durable protection against diverse pathogens.

Conflict of interest: The authors have declared that no conflict of interest exists.

Copyright: (c) 2020, American Society for Clinical Investigation.

Submitted: January 22, 2020

Accepted: April 15, 2020

Published: May 21, 2020

Reference information: /CI Insight. 2020;5(10):e136653.

https://doi.org/10.1172/jci.

insight.136653.

\section{Introduction}

Engineered nanoparticles present an exciting opportunity in vaccine design allowing for the precise manipulation of antigen presentation and targeting. The immune system has evolved to respond strongly to repetitive antigens displayed on nanoscale structures $(1-1000 \mathrm{~nm})$, the size range of most infectious microorganisms (1). Previously, spontaneously forming virus-like particles have been harnessed for highly protective vaccines against human papilloma virus, hepatitis B, and hepatitis E (2). However, because only selected pathogens spontaneously form virus-like particles, engineered nanoparticles offer an opportunity to combine the potent vaccine immunogenicity of virus-like particles with a favorable safety profile for human use.

Engineered nanoparticles carrying vaccine antigens have demonstrated superior immunogenicity over traditional subunit and whole-organism vaccines in experimental disease models of HIV (3), malaria (4), respiratory syncytial virus (5), and tuberculosis (6). For influenza, the self-assembling ubiquitous iron storage protein ferritin has been engineered into protein nanoparticle vaccines, consisting of a ferritin core with 8 trimeric influenza hemagglutinin (HA) spikes on the surface, termed HA-ferritin (7). Vaccination with HA-ferritin in mice and ferrets resulted in serum antibodies of greater magnitude, potency, and breadth compared with those elicited by the licensed inactivated influenza virus vaccine. 
Prototypic candidate vaccines of this kind have now been evaluated in human phase I clinical trials (ClinicalTrials.gov NCT03186781 and NCT03814720). However, the immune mechanisms that underpin the enhanced immunity of nanoparticle-based vaccine platforms are poorly defined (8). Previous studies have demonstrated that compared with monomeric antigens, nanoparticle loading results in improved antigen drainage to lymph nodes (LNs) (9), increased uptake and presentation by follicular dendritic cells (FDCs) (10), and greater induction of follicular helper $\mathrm{T}$ cells $\left(\mathrm{T}_{\mathrm{FH}}\right.$ cells) (11) and germinal center (GC) responses (9). Recently, the dense glycosylation of HIV envelope antigens displayed on a ferritin nanoparticle was shown to augment immunogenicity through innate recognition via the mannose-binding lectin (MBL) pathway of complement activation (12). However, deglycosylation of the antigen, or the use of MBL-knockout mice, did not completely abrogate the enhanced immunogenicity of nanoparticle delivery, suggesting additional mechanisms contribute to immune enhancement.

In this study, we contrast immune responses to a prototypic ferritin nanoparticle displaying HA versus a formulation of free HA protein analogous to current subunit vaccines. We confirmed enhanced serum antibody titers and GC formation elicited by HA-ferritin, facilitating protective immunity against high-dose influenza challenge. High-dimensional whole-organ imaging demonstrated that antigen deposition and localization in LNs were highly dissimilar between the 2 immunogens. The effect of GC enhancement by HA-ferritin on B cell receptor (BCR) mutation and the role of $\mathrm{T}_{\mathrm{FH}}$ cells were also examined. Last, we report on the immunogenicity of HA-ferritin in a nonhuman primate model by interrogation of serum antibodies and $\mathrm{LN} B$ cell and $\mathrm{T}_{\mathrm{FH}}$ responses.

\section{Results}

Expression of ferritin-based nanoparticles displaying HA from mouse-adapted influenza strain PR8. Ferritin nanoparticles expressing HA from the mouse-adapted influenza strain A/Puerto Rico/08/1934 (PR8) were produced as previously described (7). A gene encoding the ectodomain of PR8 HA was genetically fused to the ferritin gene from Helicobacter pylori, expressed using mammalian cell culture and purified using affinity and size exclusion chromatography (Supplemental Figure 1, A and B; supplemental material available online with this article; https://doi.org/10.1172/jci.insight.136653DS1). Expression and self-assembly of particles were confirmed by SDS-PAGE and transmission electron microscopy (TEM) (Supplemental Figure 1, C and D). HA spikes could be visualized protruding from the ferritin core, and the estimated size of about $37 \mathrm{~nm}$ was confirmed. Antigenicity of the HA displayed on the ferritin nanoparticles was confirmed intact, based upon binding to monoclonal antibodies reactive against the globular head (TN1F11, 441D6, TN1B09) and stem (CR9114, C179) domains of H1N1 HA (Supplemental Figure 1E). Taken together, PR8 HA-ferritin nanoparticles self-assemble with the native trimeric influenza HA structurally intact.

HA-ferritin elicits enhanced humoral immunity versus soluble antigen. Consistent with previous reports using alternative HA antigens (7), PR8 HA-ferritin nanoparticles were highly immunogenic in mice. C57BL/6 mice were vaccinated intramuscularly with high ( $3.8 \mu \mathrm{g}$ HA equivalent) or low ( $0.38 \mu \mathrm{g}$ HA equivalent) doses of HA-ferritin nanoparticles or soluble HA, both adjuvanted with AddaVax. Following a single immunization, significantly higher titers of HA-specific serum antibodies were observed for both the high ( 6 -fold) and low vaccine doses ( $\sim 32$-fold) of HA-ferritin compared with controls immunized with an equivalent dose of soluble HA (Figure 1A). Notably, enhanced immunogenicity from HA-ferritin was conserved following DNA vaccination of mice (Figure $1 \mathrm{~B}$ ), suggesting antigen-loaded nanoparticles remain intrinsically more immunogenic than free antigen whether delivered as exogenous formulations or after de novo generation in vivo.

The protective efficacy of a single vaccination with HA-ferritin nanoparticles versus soluble HA was assessed using intranasal challenge of homologous (PR8) and heterologous (influenza virus A/California/07/2009; CA09) H1N1 influenza viruses (Figure 1C). A single vaccination with either high or low doses of HA-ferritin provided complete protection against low-dose (100 50\% tissue culture infectious dose $\left.\left[\mathrm{TCID}_{50}\right]\right)$, intermediate-dose $\left(500 \mathrm{TCID}_{50}\right)$, and high-dose challenge $\left(2000 \mathrm{TCID}_{50}\right)$ with homologous PR8 virus. In contrast, immunization with soluble HA provided inferior protection, with animals susceptible at intermediate (low-dose soluble HA group) and high (both low and high soluble HA groups) challenge doses. No evidence of cross-strain protection was observed following heterologous challenge with H1N1 CA09. Therefore, vaccination with HA-ferritin nanoparticle vaccines demonstrates superior immunogenicity, dose-sparing effect, and increased protective efficacy.

$H A$-ferritin vaccination drives enhanced antigen-specific $G C$ reactions. The $\mathrm{LNs}$ draining the site of vaccine administration are a key site for the development of protective adaptive immune responses. 
A

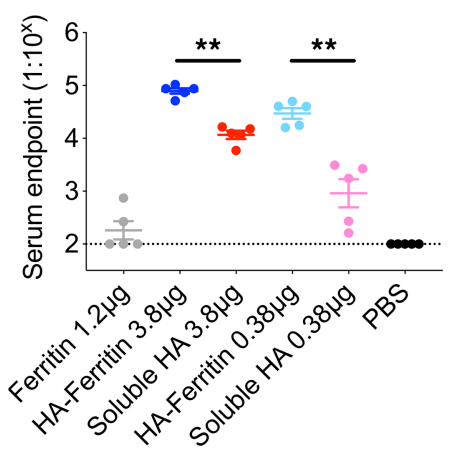

C
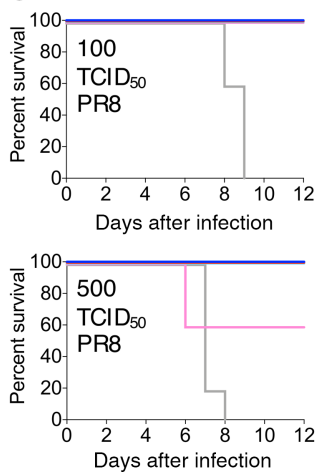

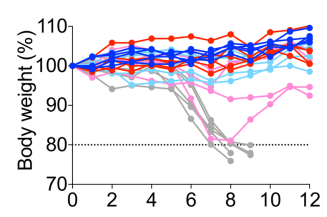

Days after infection

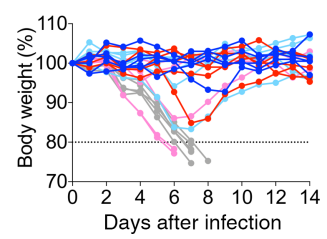

B

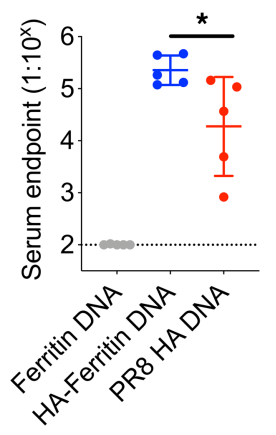

- HA-Ferritin 3.8ug

- Soluble HA $3.8 \mu \mathrm{g}$

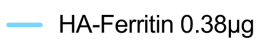

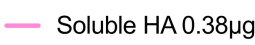

- Ferritin $1.2 \mu \mathrm{g}$

Figure 1. Vaccination with HA-ferritin nanoparticles elicits enhanced antibody titers and protective immunity. C57BL/ 6 mice ( $n=5$ mice per group) were vaccinated intramuscularly with high or low doses of HA-ferritin $(5$ or $0.5 \mu \mathrm{g})$ or a molar equivalent of soluble HA (3.8 or $0.38 \mu \mathrm{g})$. Control groups received ferritin alone $(1.2 \mu \mathrm{g})$ or PBS. All vaccines except PBS were adjuvanted with AddaVax. (A) HA-specific serum IgC titers were measured by ELISA 14 days after vaccination. Data are representative of 1 of 2 independent experiments. The dashed line indicates detection cutoff (1:100 dilution). (B) HA-specific serum IgG titers 14 days after final vaccination in mice vaccinated 3 times at 14 -day intervals with $100 \mu \mathrm{g}$ DNA encoding HA-ferritin, soluble HA, or ferritin. (C) Body weight and survival of mice immunized once 14 days before intranasal challenge with PR8 or CA09 influenza strains. The dashed line indicates $20 \%$ weight loss. Data represent mean $\pm S D$. ${ }^{*} P<0.05$, and ${ }^{* *} P<0.01$, determined by a Mann-Whitney $U$ test.

In particular, GCs facilitate somatic hypermutation and affinity maturation of antigen-specific B cells and drive the production of plasma cells secreting high-affinity antibodies. To investigate how nanoparticle vaccination affects GC induction, we first visualized draining LNs in immunized mice 14 days after intramuscular vaccination. Using the GC marker GL7, we observed extensive GC formation following HA-ferritin vaccination compared with limited GCs observed in soluble HA-vaccinated mice (Figure 2A; Supplemental Figure 2). The magnitude and longevity of GC responses in the draining LNs were assessed by flow cytometry. HA-ferritin-immunized animals displayed higher frequencies of GC B cells (IgD-B220 $0^{+} \mathrm{GL} 7^{+} \mathrm{CD} 38^{\mathrm{l}}$ ) in both draining inguinal and iliac $\mathrm{LNs}$ compared with animals vaccinated with the equivalent dose of soluble $\mathrm{HA}$, with these higher relative frequencies maintained over time out to 56 days postimmunization (Figure 2B; gating in Supplemental Figure 3). The antigen specificity of GC B cells was assessed using recombinant PR8 HA probes as previously described $(13,14)$ (representative staining in Supplemental Figure 3). At 7, 14 (Supplemental Figure 4, A and B), and 56 days after vaccination (Figure 2C), counts of PR8 HA-specific B cells in the GC were significantly or trending higher following low-dose immunization with HA-ferritin compared with soluble HA. Following high-dose vaccination, the counts of PR8 HA-specific B cells were significantly higher in HA-ferritin-vaccinated mice at day 56 but not at day 7 or 14. Therefore, immunization with HA-ferritin drives enhanced GC formation and maintenance, facilitating extended residency of HA-specific B cells within the draining LNs.

HA-ferritin immunization drives increased somatic mutation of HA-specific B cells. Extended GC reactions may facilitate increased somatic mutation of antigen-specific BCRs. To compare the ability of HA-ferritin and soluble HA vaccines to drive maturation of B cell responses, we sequenced heavy chain immunoglobulin genes from HA-specific B cells within the GCs of draining LNs (day 14 or 28 after vaccination) 
A
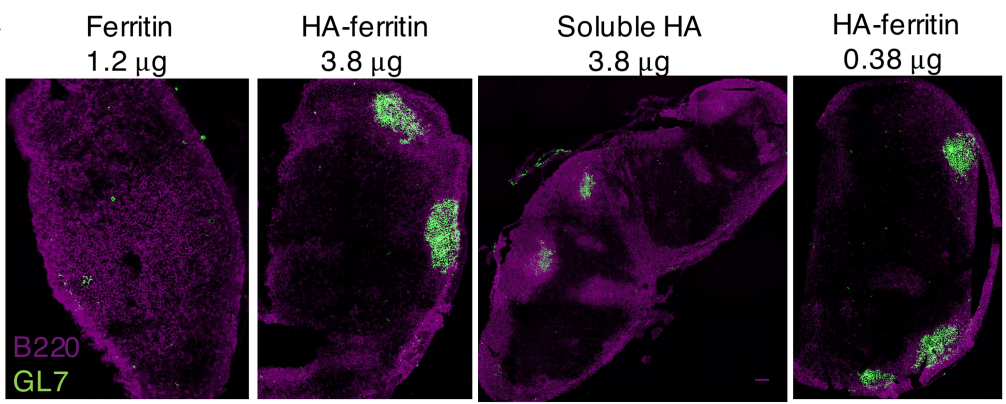

Soluble HA $0.38 \mu \mathrm{g}$
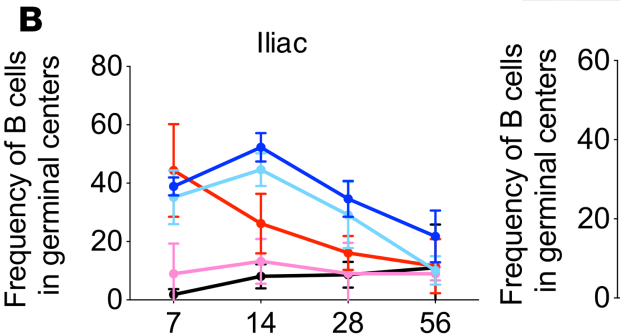

Inguinal

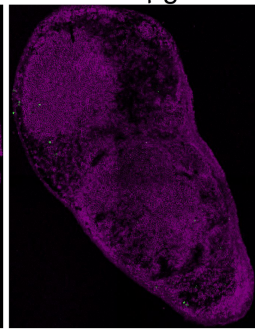

Days after vaccination
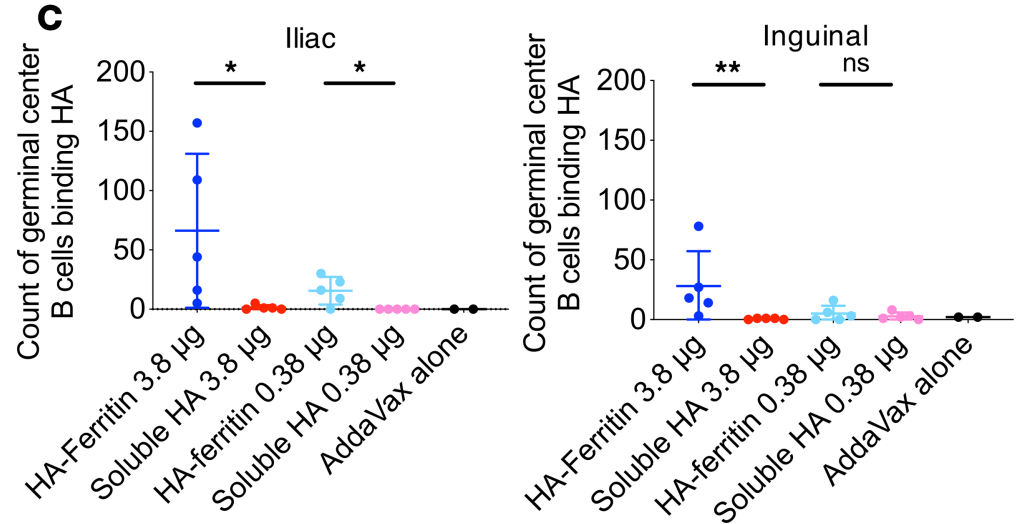

Figure 2. Augmented HA-specific GC responses in the draining LN following HA-ferritin vaccination. (A) C57BL/6 ( $n=5$ mice per group) mice were immunized with $\mathrm{HA}$-ferritin ( 5 or $0.5 \mu \mathrm{g}$ ) or a molar equivalent of soluble $\mathrm{HA}$ ( 3.8 or $0.38 \mu \mathrm{g}$ ) or $1.2 \mu \mathrm{g}$ ferritin alone, adjuvanted with AddaVax. After 14 days, draining inguinal LNs were sectioned and stained for GCs (GL7 shown in green and B220 shown in magenta). Images are representative of each treatment group. (B) Mice were vaccinated as described for $\mathbf{A}$ except for AddaVax-alone group $n=2$. The proportion of IgD-B220+ cells expressing GL7 in draining iliac (left) and inguinal (right) LNs was quantified by flow cytometry at 7, 14, 28, or 56 days after vaccination. (C) The absolute count of GC B cells (B220+IgD-CL7+) in draining iliac (left) and inguinal (right) LNs binding HA at 56 days after vaccination was measured using a probe of biotinylated PR8 HA labeled with streptavidin-PE. Data represent mean $\pm S D$ and are representative of 1 of 2 independent experiments. ${ }^{*} P<0.05$, and ${ }^{* *} P<0.01$, determined by a Mann-Whitney $U$ test.

and memory B cells ( $\left.\mathrm{IgD}^{-} \mathrm{GL} 7^{-} \mathrm{CD} 38^{+}\right)$isolated from the blood and spleen (day 71 after vaccination). The extent of variable gene mutation in HA-specific B cells was comparable in GCs from both HA-ferritin- and soluble HA-vaccinated mice at both day 14 (mean $2.32 \%$ vs. $2.17 \%$ ) and day 28 (3.56\% vs. $3.57 \%$ ) (Figure $3 \mathrm{~A})$. However, within the HA-specific memory B cell population, the degree of somatic mutation observed was significantly elevated following HA-ferritin immunization compared with soluble HA (mean $4.08 \%$ vs. 2.83\%) (Figure 3B). Therefore, augmented GC activity following HA-ferritin immunization may facilitate increased maturation of antigen-specific B cells, potentiating both quantitative and qualitative improvements to the serological response.

Retention and focused deposition of antigen in GCS following HA-ferritin vaccination. The capacity of antigen-associated glycan to drive interaction with MBL and consequent deposition of ferritin nanoparticles into GCs was recently reported (12). Using lightsheet microscopy, we similarly observed greater accumulation of fluorescently labeled HA-ferritin (Figure 4A; Supplemental Video 1) within draining LNs compared with soluble antigen (Figure 4B; Supplemental Video 2). At 14 days after vaccination, nanoparticles were colocalized with $\mathrm{CD}^{2} 5^{+} \mathrm{FDCs}$ and GCs, identified as pockets of $\mathrm{IgD}^{-}$cells surrounded by $\mathrm{IgD}^{+} \mathrm{B}$ cells. In contrast, 
A

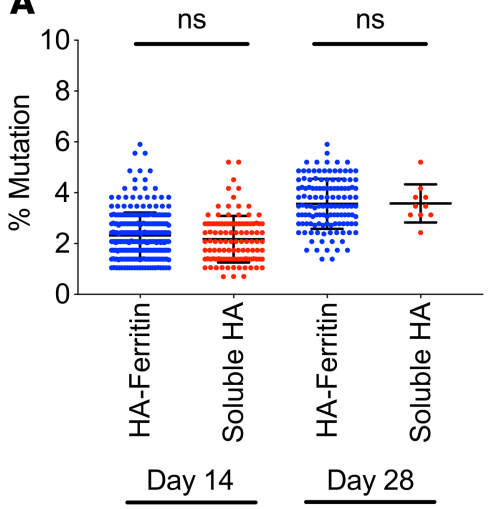

B

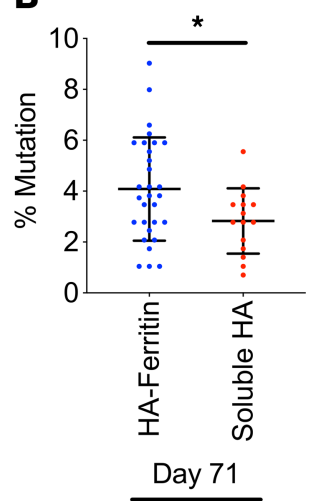

Figure 3. Increased mutation in immunoglobulin genes of memory $B$ cells following HA-ferritin vaccination. C57BL/6 $(n=6$ mice per group) mice were immunized with HA-ferritin $(5 \mu \mathrm{g})$ or a molar equivalent of soluble HA (3.8 $\mu \mathrm{g})$, both adjuvanted with AddaVax. (A) HA-specific GC B cells in draining LNs at days 14 and 28 after vaccination or (B) memory B cells in the spleen and blood at day 71 after vaccination were single cell sorted by FACS. Immunoglobulin heavy chain variable domain genes were amplified and sequenced. The mutation rate from germline murine variable domain sequences was determined. Data represent mean \pm SD of $10-270$ cells. ${ }^{*} P<0.05$, determined by a Mann-Whitney $U$ test.

GC accumulation of soluble HA antigen was not observed in mice vaccinated with soluble HA, suggesting that focused deposition of antigen underpins augmented GC function following nanoparticle immunization.

Ferritin-specific $T_{F H}$ responses are minimal. $\mathrm{T}_{\mathrm{FH}}$ cells are antigen-experienced $\mathrm{CD} 4^{+} \mathrm{T}$ cells that drive the formation and maintenance of GCs $(15,16)$. Enhanced GC formation following HA-ferritin nanoparticle immunization could potentially be mediated via a differential capacity to recruit $\mathrm{T}$ cell help. In particular, $\mathrm{T}$ cells specific for epitopes within the Helicobacter ferritin core could assist in activation of HA-specific B cells via linked recognition (intermolecular help) (17). The proportion of $\mathrm{T}_{\mathrm{FH}}$ cells $\left(\mathrm{CD} 4^{+} \mathrm{CXCR} 5^{+} \mathrm{PD}-1^{+}\right)$ in the draining iliac or inguinal LNs, as enumerated by flow cytometry, was similar in animals vaccinated with either soluble HA or HA-ferritin (Figure 5A, gating in Supplemental Figure 5), as were the absolute counts (Supplemental Figure 6). The antigen specificity of $\mathrm{T}_{\mathrm{FH}}$ cells was examined using an activation-induced marker assay (18) following restimulation of LN homogenates with pools of overlapping peptides spanning either ferritin or HA proteins. Activation of $\mathrm{T}_{\mathrm{FH}}$, measured using the canonical $\mathrm{B}$ cell helper ligand CD154 (Figure 5B, gating in Supplemental Figure 7), or activation markers CD25 and OX40 (Figure $5 \mathrm{C}$ ), was not observed in response to stimulation with ferritin peptides. In contrast, stimulation with HA peptides elicited $\mathrm{T}_{\mathrm{FH}}$ upregulation of CD25 and OX40 in all groups vaccinated with either soluble HA or HA-ferritin. CD154 expression was increased in both high-dose groups and low-dose HA-ferritin but not low-dose soluble HA. These data suggest that linked recognition of ferritin-specific $\mathrm{T}_{\mathrm{FH}}$ cells by HA-specific $B$ cells is not significant, but increased $\mathrm{T}_{\mathrm{FH}}$ function may track with the augmented immunogenicity of HA-ferritin at lower vaccine doses.

Lack of HA stem response suggests direct interaction of $H A$-ferritin with $B$ cells in vivo. The capacity for HA-ferritin to drive the efficient cross-linking of HA-specific BCRs and consequent B cell activation has been established in vitro $(19,20)$. However, B cell signaling is difficult to directly observe in vivo, and it remains unclear if intact nanoparticles comprise a significant proportion of the immunogenic material interacting with B cells following immunization. We therefore used indirect serological measures to examine the comparative immunogenicity of the 2 domains of HA following HA-ferritin immunization: the nanoparticle-distal globular head and the nanoparticle-proximal stem domain. We hypothesized that direct interaction of B cells with intact nanoparticles displaying the HA array would favor head-specific responses, while the stem-specific responses would remain low or absent because the stem domain is less accessible by BCR binding. This is in contrast to soluble HA, where both the head and stem domains are readily accessible to BCR binding. High-dose soluble HA vaccination elicited serum IgG against stem (Figure 6A) and fulllength HA (Figure 6B) detectable from 14 days postvaccination. In contrast, HA-ferritin at both high and low doses elicited negligible serum IgG production against the HA stem despite strong serological responses against full-length HA elicited from 7 days postvaccination. Therefore, HA loading onto nanoparticles mirrors the steric constraints of HA trimers on influenza virions (21) and suggests that structurally intact nanoparticles are directly recognized by B cells in vivo.

Enhanced immunogenicity of HA-ferritin is not observed in pigtail macaques. We investigated whether the enhanced immunogenicity of HA-ferritin observed in mice translated to nonhuman primate immunization models. Pigtail macaques were intramuscularly immunized with a single dose of $15 \mu \mathrm{g}$ HA-ferritin or an equimolar amount of soluble HA, adjuvanted with AddaVax. Serum IgG responses against full-length HA were 


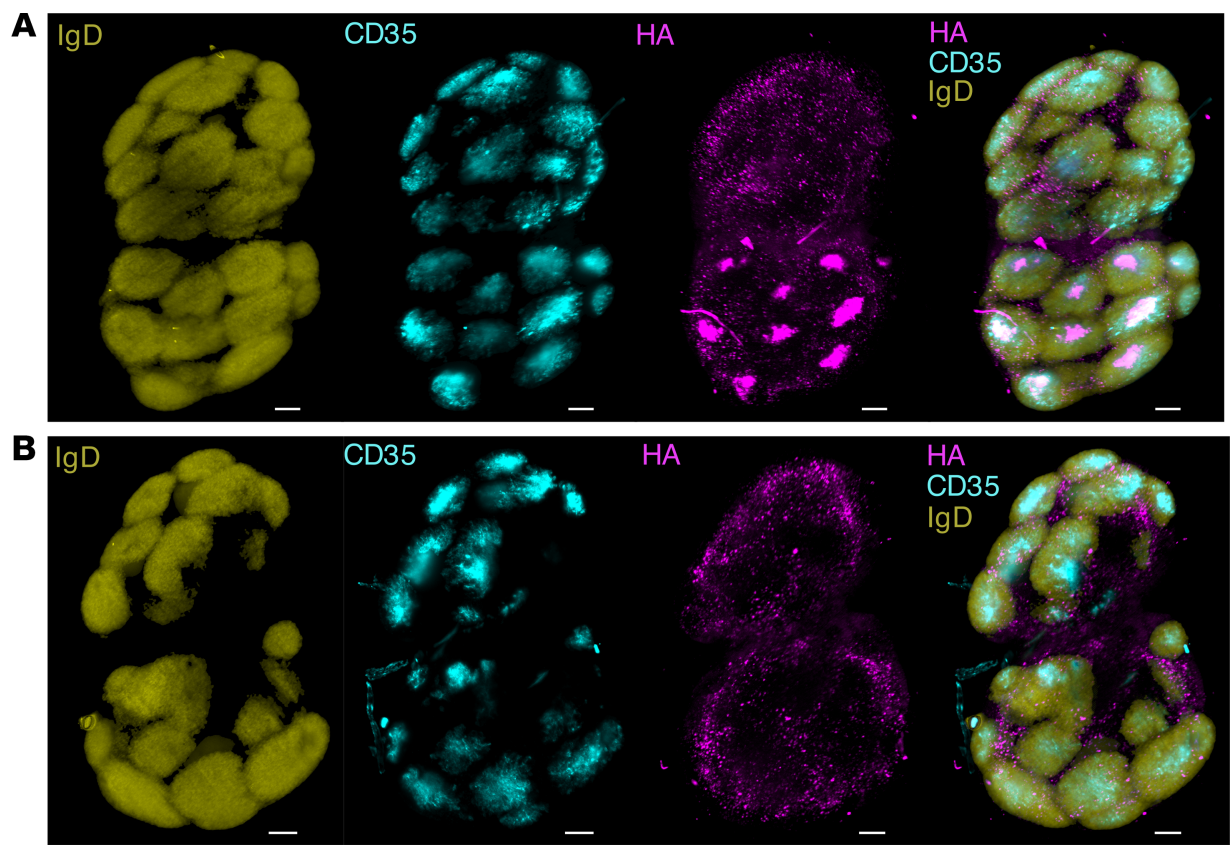

Figure 4. Increased deposition of HA-ferritin in GCs. C57BL/6 mice ( $n=2$ mice per group) were immunized with Alexa Fluor 647-labeled HA-ferritin (5 $\mu \mathrm{g}$ ) (A) or a molar equivalent of Alexa Fluor 647-labeled soluble HA (3.8 $\mu \mathrm{g})$ (B), adjuvanted with AddaVax. After 14 days, draining inguinal LNs were stained (IgD, to identify B cells, yellow; CD35, a marker of FDCs, blue), cleared and imaged by lightsheet microscopy. Images are maximum intensity projections of Z-stacks and are representative of each treatment group. Scale bar: $200 \mu \mathrm{m}$

comparable between the 2 groups at 21 days after vaccination (Figure 7A). Stem HA antibodies were similarly low between the 2 vaccine groups (Supplemental Figure 8A). The HA inhibition (HAI) titers against PR8 virus at 21 days after vaccination were generally low, although they did appear higher in 3 of 5 HA-ferritin-vaccinated animals (Supplemental Figure 8B). $\mathrm{T}_{\mathrm{FH}}$ and GC B cell responses in draining LNs were examined at 21 days postvaccination. No significant changes in the frequency of $\mathrm{T}_{\mathrm{FH}}$ cells $\left(\mathrm{CD} 4^{+} \mathrm{CXCR} 5^{+} \mathrm{PD}-1^{+}\right)$(Figure 7B, gating in Supplemental Figure 9) or the proportion of $\mathrm{T}_{\mathrm{FH}}$ cells expressing transcription factor Bcl6 and proliferation marker Ki-67 (Figure 7C) were observed between draining LNs versus nondraining axillary LNs in either HA-ferritin or soluble HA groups. Similarly, the frequency of GC B cells $\left(C D 20^{+} \operatorname{IgM}-\operatorname{IgD}-\operatorname{IgG}{ }^{+} \mathrm{Bcl} 6^{+} \mathrm{Ki}^{-} 67^{+}\right)$ was generally unchanged following vaccination with either HA-ferritin or soluble HA (Figure 7D, gating in Supplemental Figure 10). Notably, HA-specific GC B cells were detected in the draining LNs of 3/5 animals vaccinated with HA-ferritin versus $0 / 5$ animals immunized with soluble HA (Figure 7E, representative staining in Supplemental Figure 10). Overall, while a proportion of pigtail macaques showed increased frequencies of HA-specific GC B cells upon HA-ferritin immunization, we did not observe any significant enhancement in serum IgG, GC, or $\mathrm{T}_{\mathrm{FH}}$ responses compared with animals vaccinated with soluble $\mathrm{HA}$.

\section{Discussion}

Numerous nanoparticle-based vaccines are currently in clinical development as improved immunization platforms against infectious diseases (reviewed in ref. 8). However, the precise immune mechanisms underpinning the enhanced immunogenicity of many particulate vaccines remain unclear. Previously, self-assembling HA-ferritin nanoparticles were shown to augment neutralizing and cross-reactive antibody responses against influenza virus in ferret and mouse models (7). Similarly, we find ferritin nanoparticle display of HA from PR8 dramatically increased HA-specific serum antibody titers and consequent protective efficacy in mice compared with immunization with free HA equivalents. In addition, we demonstrate augmented and longer-lasting GC reactions within draining LNs, focused deposition of antigen in GCs of draining LNs, and greater BCR mutation in memory B cells following HA-ferritin immunization but no improvement in HA-specific $\mathrm{T}_{\mathrm{FH}}$ responses. The improved serological outcomes elicited by ferritin nanoparticle vaccines in our murine model appear linked to the capacity to drive greater involvement of antigen-specific B cells in GCs, facilitating improved affinity maturation and/or expanded plasma cell differentiation. 

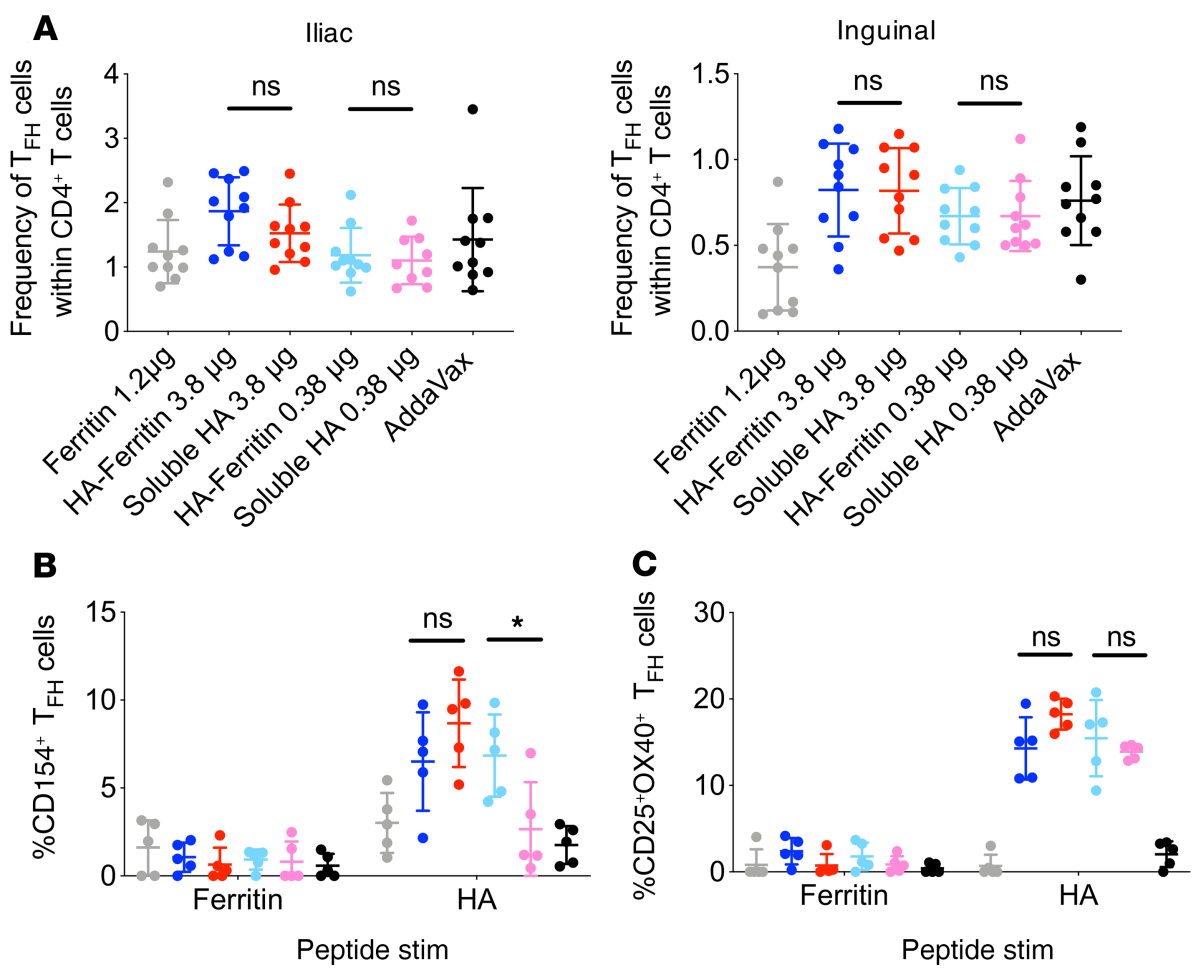

Figure 5. Superior immunity of HA-ferritin is not driven by ferritin-specific $\mathbf{T}_{\mathrm{FH}}$ cells. (A) C57BL/6 ( $n=10$ mice per group) mice were immunized with HA-ferritin ( 5 or $0.5 \mu \mathrm{g}$ ) or a molar equivalent of soluble HA (3.8 or $0.38 \mu \mathrm{g}$ ), or $1.2 \mu \mathrm{g}$ ferritin alone, adjuvanted with AddaVax. Fourteen days following vaccination, draining iliac (left) and inguinal (right) LNs were harvested. The proportion of $\mathrm{T}_{\mathrm{FH}}$ cells (identified as CXCR5+PD-1+) within the $C D 4^{+} \mathrm{T}$ cell population was determined by flow cytometry. Data shown are combined from 2 independent experiments. Fourteen days after mice were vaccinated as in $\mathbf{A}$, inguinal and iliac $L N s$ were harvested and pooled for each mouse ( $n=5$ mice per group). Lymph node suspensions were cultured for 18 hours with pools of overlapping peptides spanning HA or ferritin proteins (final concentration of each peptide, $2 \mu \mathrm{g} / \mathrm{mL}$ ) or DMSO alone. Antigen specificity was determined by the proportion of $\mathrm{T}_{\mathrm{FH}}$ cells positive for ligand CD154 (B) or activation markers $\left(C D 25^{+} 0 \times 40^{+}\right)($C) following subtraction of DMSO background. Data represent mean $\pm \mathrm{SD}$. ${ }^{*} P<0.05$, determined by a Mann-Whitney $U$ test.

Critically, the heightened immunogenicity imparted by HA-ferritin was not recapitulated in immunized nonhuman primates, highlighting the potential technical and immunological complexities when extrapolating data from murine studies to higher-order primate models.

Other studies have reported increased GC activity following nanoparticle vaccination (10, 12, 22). A number (or combination) of discrete mechanisms could contribute to this effect. The high antigenic density and particulate nature of nanoparticle vaccines can facilitate increased antigen delivery to draining LNs following immunization, increasing both spatial and temporal opportunities for activation of naive B and T cells and the initiation of GC reactions. Coating with natural $\operatorname{IgM}(9,23)$ or complement activation via classical (24), alternative (25), and MBL (12) pathways can increase retention of nanoparticles by GC-resident FDCs. Here, we showed concentration of HA-ferritin in draining LNs with GC-resident FDCs, which we failed to detect for soluble HA. Previously, glycosylation patterns on ferritin-based nanoparticles were shown to be critical for immunogenicity and concentration in GCs (12). However, complete deglycosylation of ferritin particles reduced the serum IgG response by only 2- to 3-fold, suggesting other factors are also important. In the present study, both soluble HA and HA-ferritin were expressed using the same mammalian Expi293 cells, presumably allowing comparable glycosylation patterns. Further, that the superior immunogenicity of HA-ferritin over soluble HA is maintained following DNA vaccination suggests factors beyond glycosylation are likely to contribute.

The activity of antigen-specific $\mathrm{T}_{\mathrm{FH}}$ cells could contribute to augmented GC function. We find that despite increased frequencies of GC B cells following HA-ferritin vaccination, the proportion of $\mathrm{T}_{\mathrm{FH}}$ cells was comparable after vaccination with either HA-ferritin or soluble HA antigen. This is consistent with other reports using $\sim 40$-nm self-assembling protein nanoparticles (12) and $\sim 25$-nm virus-like particles (26) 

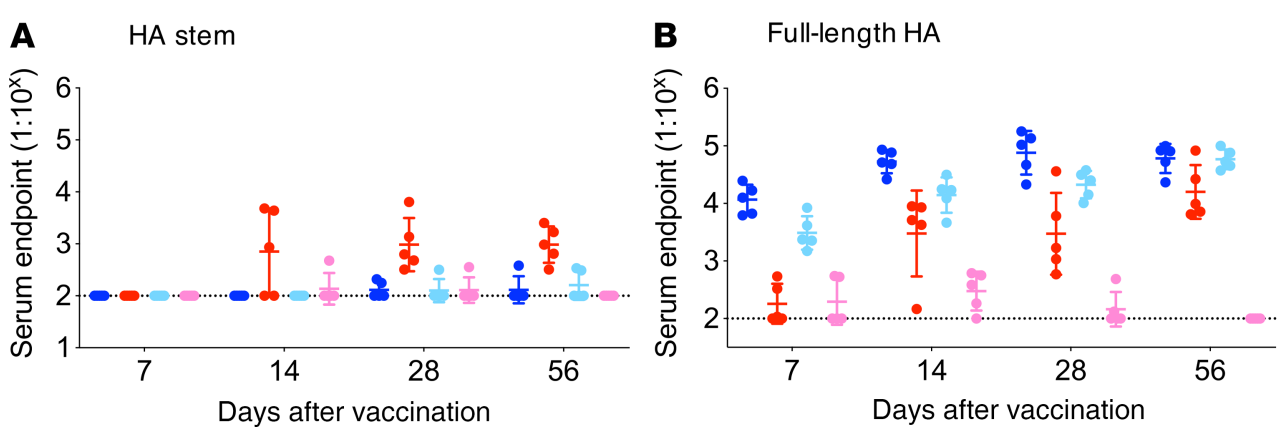

- $3.8 \mu \mathrm{g} \mathrm{HA-Ferritin}$

- $3.8 \mu \mathrm{g}$ Soluble HA

- $0.38 \mu \mathrm{g} \mathrm{HA}$-Ferritin

$0.38 \mu \mathrm{g}$ Soluble HA

Figure 6. HA-stem response hindered by particulate display of antigen. C57BL/ 6 mice ( $n=5$ mice per group) were vaccinated intramuscularly with high or low doses of HA-ferritin ( 5 or $0.5 \mu \mathrm{g}$ ) or a molar equivalent of soluble HA (3.8 or $0.38 \mu \mathrm{g})$. All vaccines were adjuvanted with AddaVax. Serum lgG titers against stem HA (A) or full-length HA (B) were measured by ELISA at 7, 14, 28, or 56 days after vaccination. Data represent mean \pm SD and are representative of 1 of 2 independent experiments. The dashed line indicates detection cutoff (1:100 dilution).

but in contrast to the $\mathrm{T}_{\mathrm{FH}}$ expansion observed following vaccination with antigen conjugated to $\sim 200-\mathrm{nm}$ lipid vesicles $(11,22)$ or 130-nm synthetic poly(lactic-co-glycolic acid) particles (4). In terms of specificity, we detected few $\mathrm{T}_{\mathrm{FH}}$ cells specific for the Helicobacter ferritin core, suggesting that linked recognition of ferritin plays a small or no role in supporting increased HA-specific B cell responses following immunization with HA-ferritin in our model. In response to HA peptides, expression patterns of activation markers CD25 and OX-40 were largely consistent between vaccine groups, while CD154 expression was substantially higher following low-dose HA-ferritin vaccination compared with soluble HA. Costimulatory molecule CD154 (CD40L) directly engages CD40 on B cells, promoting maintenance of established GC responses (27). Therefore, at low doses, HA-ferritin vaccines may maintain a superior capacity to promote $\mathrm{T}_{\mathrm{FH}}$ activation and the provision of $\mathrm{T}$ cell help.

Multimeric $B C R$ engagement via direct interaction of intact nanoparticles with $B$ cells contributes to potent B cell activation and consequent GC differentiation (28). The ability of HA-ferritin nanoparticles to potently stimulate $\mathrm{BCR}$ cross-linking in vitro has previously been demonstrated using engineered $\mathrm{B}$ cell lines expressing HA-specific BCR $(19,29)$, an effect not observed with free HA protein alone (20). However, BCR engagement and signaling are difficult to demonstrate in vivo, as is determining that antigen-loaded nanoparticles are conformationally intact upon presentation to B cells in draining LNs. As an indirect measure of this, we assessed the comparative immunogenicity of the 2 domains of HA, the globular head comprising the trimer apex and the C-terminal stem domain. We find in comparison with free HA trimers, epitopes in the HA stem are poorly targeted in the polyclonal antibody response following HA-ferritin immunization. This is consistent with other studies where HIV envelope (Env) antigen loaded onto nanoparticles sterically blocks epitopes located proximal to the nanoparticle surface, reducing antigenicity in vitro (30) and immunogenicity in vivo $(30,31)$. In the present study, the serological evidence indirectly suggests that naive B cells are primed by intact nanoparticles in vivo. Further in-depth interrogation of nanoparticle-B cell interactions in LNs is warranted, likely requiring the use of technologies such as Nur77-EGFP transgenic mice (32) or similar as sensors of BCR signaling in vivo. In the case of influenza vaccination, a reduced immune response to the highly conserved HA stem may limit the potential of a nanoparticle as a "universal" vaccine. This necessitates creative solutions to antigen display, such as nanoparticles displaying only stabilized stem (29).

Although ferritin-based nanoparticle vaccines are highly protective in rodent models (7), there have been few studies that have advanced into large and/or outbred animal models. HA-ferritin conferred greater protection than standard trivalent inactivated vaccines (TIVs) in a ferret model of influenza infection, although the antigenic integrity and glycosylation of HA in TIV are unclear (7). There are mixed results in the context of HIV. Ferritin nanoparticles displaying HIV Env trimers do appear more immunogenic than soluble trimers in rabbits immunized using a DNA prime-protein boost approach, though less pronounced compared with mice (33). However, alternative Env immunogens (ConM SOSIP) on ferritin show no advantage over soluble trimers in terms of serum antibody induction in rabbits (30), although there is some evidence of increased early antibody responses following priming (34). HIV-ferritin nanoparticles are clearly immunogenic in rhesus macaques (34) although lacking a direct comparison to soluble protein. Immunization of cynomolgus macaques with ferritin nanoparticles displaying EpsteinBarr virus antigen shows superior immunogenicity compared with the soluble antigen counterpart $(35,36)$. 
A

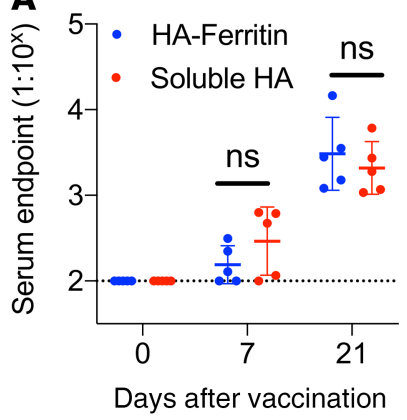

B

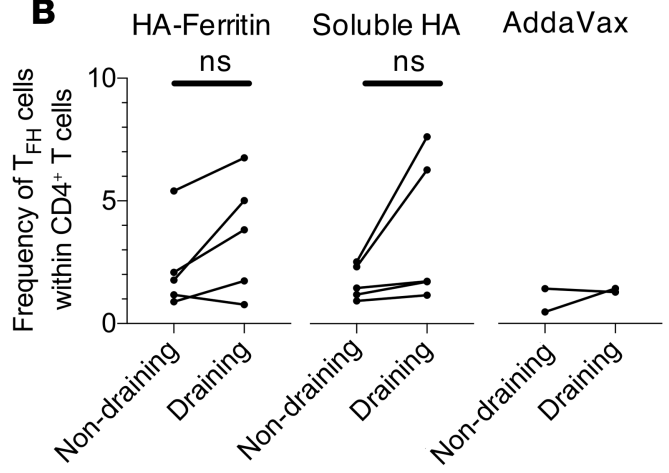

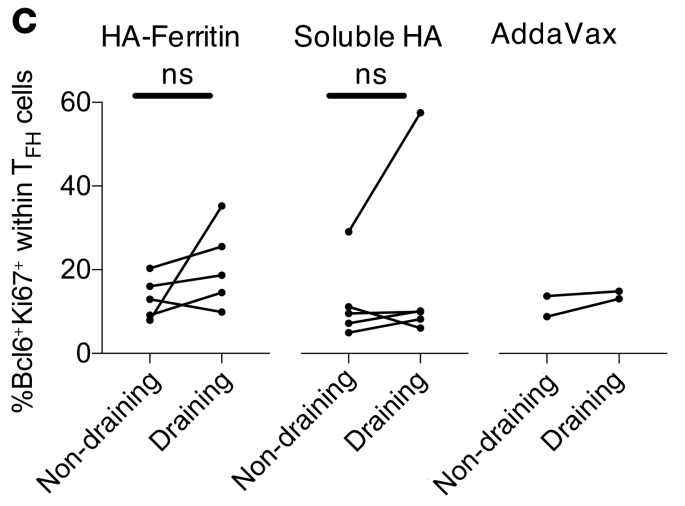

D

HA-Ferritin

Soluble HA

AddaVax
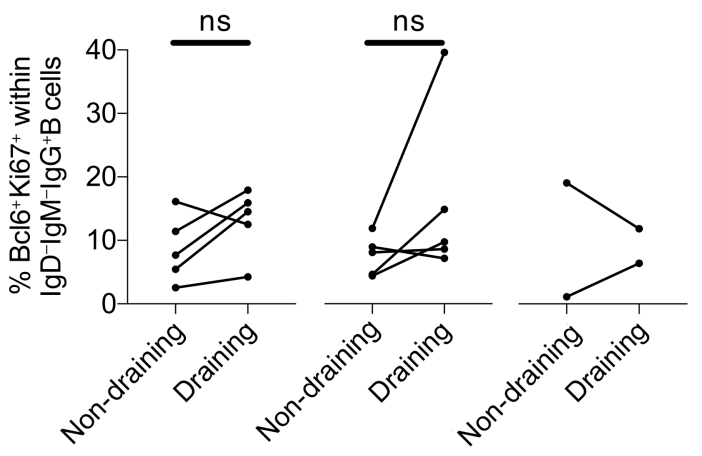

\section{AddaVax}
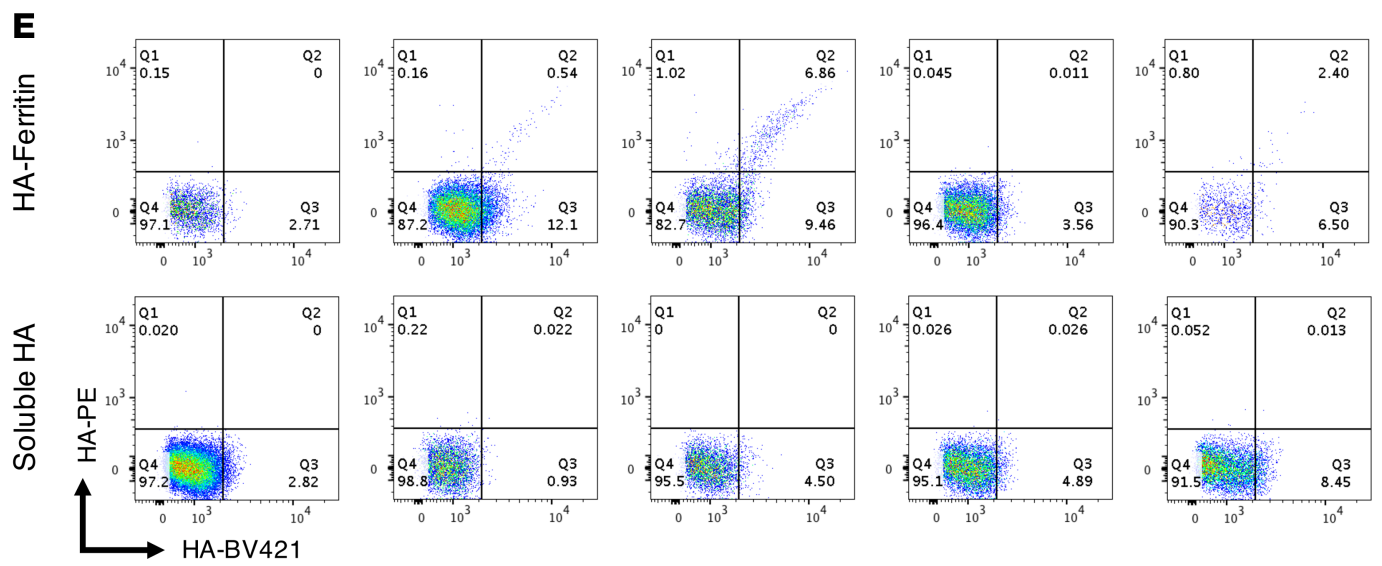

Figure 7. Enhanced immunogenicity of HA-ferritin over soluble HA does not translate to nonhuman primates. Pigtail macaques $(n=5)$ were vaccinated once intramuscularly with HA-ferritin $(15 \mu \mathrm{g})$ or a molar equivalent of soluble HA (11.25 $\mu \mathrm{g})$, both adjuvanted with AddaVax. A control group received AddaVax alone $(n=2)$. (A) Serum IgG titers against HA were measured by capture ELISA at 0,7 , and 21 days after vaccination. The dashed line indicates detection cutoff (1:100 dilution). Data represent mean \pm SD. (B) At day 21, draining and nondraining LNs were harvested. The proportion of $\mathrm{T}_{\mathrm{FH}}$ cells (identified as CXCR5 ${ }^{+} \mathrm{PD}-1^{+}$) within $\mathrm{CD} 4^{+} \mathrm{T}$ cells and (C) their expression of transcription factor $\mathrm{Bcl} 6$ and proliferation marker Ki-67 were determined. (D) The proportion of CD20+lgM-lgD-lgG+ $B$ cells involved in LN GCs (identified as Bcl6 $\left.6^{+} \mathrm{Ki}-67^{+}\right)$. (E) Flow cytometry plots showing HA specificity of these GC B cells in draining LNs measured using dual probes of biotinylated PR8 HA labeled with streptavidin-fluorophores. Data collected from 1 experiment. Analyzed using a Mann-Whitney $U$ test (A) or Wilcoxon's matched-pairs signed-rank test (B-D).

While the differences in antigen-binding, serum antibody titers are minor, the neutralizing capacity of antibodies are vastly different, even in the context of preexisting immunity. In the present study, serum antibody titers against HA were comparable in soluble- and nanoparticle-vaccinated macaques, although HAI titers appeared modestly higher in HA-ferritin-vaccinated animals, possibly indicative of some qualitative improvements in the serological response. However, frequencies of GC B cells and $\mathrm{T}_{\mathrm{FH}}$ cells in macaque LNs were consistent between HA-ferritin- and soluble HA-immunized animals, in sharp contrast to the highly reproducible and pronounced enhancement of GC activity seen in mice. 
A number of factors could contribute to the divergent responses to HA-ferritin vaccines observed in our studies using mice and macaques. First, the pigtail macaques used in this study are an outbred population and express polymorphic MHC, BCR, and FcR alleles with unknown influences on vaccine immunogenicity. In humans, heterogeneity in MHC-II molecules that present influenza-derived peptides results in variable immunity against influenza (37). Similarly, we previously established the differential immunogenicity of the HA stem domain in BALB/c versus C57BL/6 mice may be partially a function of distinct MHC-II genetics (14). Increasing experimental group sizes in outbred animals may be necessary to observe statistically significant differences. However, given the significant costs and logistical challenges of nonhuman primate studies, these questions may ultimately best be answered by detailed analysis from human clinical trials either planned or underway (ClinicalTrials.gov NCT03186781, NCT03814720).

Second, the mechanisms of innate cell recognition of HA-ferritin in primates need further clarification. In mice, the immunogenicity of ferritin nanoparticles displaying viral antigens was recently reported to be heavily influenced by MBL recognition of glycosylated antigen (12). However, in humans, MBL is highly polymorphic, with 23 known MBL haplotypes (38), correlating with 1000-fold variations in serum MBL concentration (39). In contrast to MBL-knockout mice, which are more susceptible to influenza (40), S. aureus (41), and nematode (42) infections, adult humans with MBL deficiency do not suffer from increased morbidity or mortality from infectious diseases (43), illustrating potential redundancy in innate immune pathways in humans. Higher-order primates, including macaques, express the same MBL-2 gene as humans but lack polymorphisms and express MBL-1, a pseudogene in humans (44). It is therefore currently unclear if the dominant role of $\mathrm{MBL}$, or other critical pathways of innate immune recognition modulating nanoparticle vaccine immunogenicity in mice, is recapitulated in some or all primate species.

Third, the dose and timing of vaccination and experimental assessment may contribute to apparent vaccine immunogenicity between species. In the present study, macaques received a $15-\mu \mathrm{g}$ HA-equivalent dose, equal to the dose delivered in human seasonal influenza vaccinations. However, given macaques are naive whereas humans has extensive preexisting immunity, it is unclear if differential outcomes between soluble and nanoparticle delivery may have become apparent with higher dose immunizations or after assessment at alternative time points given cell metabolism and proliferation in macaques are slower than in mice (45). Alternatively, booster immunization may be required to more sensitively detect the differential immune outcomes of HA-ferritin versus soluble HA or could further be enhanced by modifying antigen delivery duration through osmotic pumps or escalating doses, which has been shown to enhance the magnitude and quality of $\mathrm{GC}$ and $\mathrm{T}_{\mathrm{FH}}$ responses in rhesus macaques (46). At a technical level, we note that complex lymphatic drainage patterns and clustering of LNs make accurate identification of the principal antigen draining node problematic in macaques, resulting in an underestimation of the immune readout. In rhesus monkeys, nanoparticle vaccination in the deltoid muscle drains only sporadically to the axillary LNs, while subcutaneous administration showed more consistent drainage to the axillary LNs (47), potentially allowing for more accurate quantification of GC responses in future trials.

In summary, our data suggest that within mice, increased LN deposition of HA-ferritin, and likely heightened BCR cross-linking in vivo, can drive extended GC activity that increases the quantity and quality of protective serum antibody responses. However, in nonhuman primates similarly immunized, we see no systemic enhancement of immunogenicity of a ferritin-based influenza vaccine, suggesting important unresolved differences exist between rodent and primate models of immunization. A greater mechanistic understanding into nanoparticle vaccine recognition by innate and adaptive immunity, nanoparticle vaccine trafficking and deposition in vivo, and any relevant differences that exist between animal immunization models is required to clarify the potential of nanoparticle platforms as next-generation human vaccines for influenza and other important pathogens.

\section{Methods}

Design and expression of ferritin nanoparticles. The development of recombinant ferritin and HA-ferritin nanoparticles was previously described in detail (7). Briefly, a gene encoding the ectodomain of HA from A/Puerto Rico/08/1934 modified with a Y98F substitution to eliminate sialic acid binding was fused to a modified gene encoding $H$. pylori nonheme iron-containing ferritin (GenBank NP_223316) using a SerGly-Gly linker. This fusion construct was subcloned into a mammalian expression vector. DNA was purified using EndoFree Plasmid Mega Kit (QIAGEN). Ferritin and HA-ferritin nanoparticles were expressed by transient transfection of Expi293F (Life Technologies, Thermo Fisher Scientific) suspension cultures 
and purified using ion exchange chromatography with HiTrap Q HP column (GE Healthcare) or lectin Galanthus nivalis agglutinin (EY Laboratories, Inc.), respectively. Nanoparticle preparations were further purified using size exclusion chromatography using Superose 6 PG XK 16/70 column (GE Healthcare), with correct expression confirmed by SDS-PAGE.

Transmission electron microscopy. Purified nanoparticles were negatively stained with $1 \%$ uranyl acetate (Polysciences) in Milli-Q water (MilliporeSigma), and TEM images were recorded on a FEI Tecnai TF20 instrument with an operation voltage of $200 \mathrm{kV}$.

Antigenicity ELISA. MaxiSorp plates (Nunc) were coated overnight with the purified nanoparticles. After blocking with 5\% skim milk powder in PBS, serial dilutions of human mAbs (produced in-house) specific for the head or stem of HA were added. Plates were washed before adding an HRP-conjugated anti-human IgG (P0214, DAKO) at 1:30,000. Plates were washed and developed using TMB substrate (MilliporeSigma) and read at $450 \mathrm{~nm}$.

HA protein synthesis and purification. Recombinant full-length (13) and stem-only (14) PR8 HA protein with ablated sialic acid binding were prepared as previously described and used as either flow cytometry probes or an immunogen. Briefly, HA genes were synthesized (GeneArt) and subcloned into mammalian expression vectors. HA protein was expressed by transient transfection of Expi293 (Life Technologies, Thermo Fisher Scientific) suspension cultures and purified by polyhistidine-tag affinity chromatography and gel filtration. For use as flow cytometry probes, proteins were biotinylated using BirA (Avidity) and stored at $-80^{\circ} \mathrm{C}$. Before use, biotinylated HA proteins were labeled by the sequential addition of streptavidin-conjugated to phycoerythrin (PE) (Life Technologies, Thermo Fisher Scientific) or BV421 (BD) and stored at $4^{\circ} \mathrm{C}$.

Mouse immunizations and viral challenge. All mice were female C57BL/6 aged 6-8 weeks at the start of experiments. Mice were anesthetized by isoflurane inhalation before infection or immunization. Groups of 5 mice were immunized with either 5 or $0.5 \mu \mathrm{g}$ of HA-ferritin nanoparticles (corresponding to 3.8 and $0.38 \mu \mathrm{g}$ of $\mathrm{HA}$, respectively); 3.8 or $0.38 \mu \mathrm{g}$ of soluble trimeric HA; $1.2 \mu \mathrm{g}$ ferritin nanoparticles; $100 \mu \mathrm{g}$ DNA plasmid encoding HA-ferritin fusion, HA or ferritin, or PBS. All immunizations were given intramuscularly into both hind quadriceps using a 29-gauge needle. All protein vaccines were formulated with AddaVax (InvivoGen) at a 1:1 ratio except PBS-only controls. Blood samples were collected by cardiac puncture 14 days after immunization and serum frozen. For viral challenge experiments, mice were intranasally instilled with $50 \mu \mathrm{L}$ of the specified $\mathrm{TCID}_{50}$ dose of A/Puerto Rico/08/1934 (PR8) or A/California/04/2009 (CA09). The mice were observed for clinical signs of infection and weight measurements were recorded daily.

Macaque studies. Twelve pigtail macaques (Macaca nemestrina) recycled from previous HIV vaccination studies were randomized for use in this study. Each experimental group contained 1 female with the remainder being male, aged between 21 and 36 months. Before any procedure the animals were anesthetized intramuscularly with ketamine. Animals were immunized in the right deltoid muscle with 1 dose of HA-ferritin $(15 \mu \mathrm{g})$ or a molar equivalent of soluble HA $(11.25 \mu \mathrm{g})$ formulated 1:1 v/v with AddaVax (Invitrogen, Thermo Fisher Scientific). Serial blood samples were collected and serum isolated. At necropsy 21 days after vaccination, draining and nondraining LNs were collected and homogenates were cryopreserved.

Mouse serum antibody ELISA. Overnight $2 \mu \mathrm{g} / \mathrm{mL}$ HA protein was coated onto MaxiSorp plates (Nunc). Plates were blocked with 5\% skim milk powder in PBS before adding serially diluted mouse sera in duplicate. Plates were washed before addition of an HRP-conjugated anti-mouse IgG (474-1806, KPL) at 1:10,000 dilution. Plates were washed and developed using TMB substrate (MilliporeSigma); reaction was stopped with $0.16 \mathrm{M} \mathrm{H}_{2} \mathrm{SO}_{4}$ and read at $450 \mathrm{~nm}$. Endpoint titers were calculated as the reciprocal serum dilution giving signal 2 times background using a fitted curve (4-parameter log regression).

Macaque serum antibody capture ELISA. Overnight $0.1 \mu \mathrm{g} / \mathrm{mL}$ rabbit anti-His polyclonal antibody (GenScript) was coated onto MaxiSorp plates (Nunc). Plates were blocked with 5\% skim milk powder in PBS before adding His-tagged full-length or stem HA protein. Serially diluted macaque sera were added in duplicate. Plates were washed before addition of an HRP-conjugated anti-macaque IgG (1B3, Kerafast) at 1:8000. Plates were washed and developed using TMB substrate (MilliporeSigma); reaction was stopped with 0.16 $\mathrm{M} \mathrm{H}_{2} \mathrm{SO}_{4}$ and read at $450 \mathrm{~nm}$. Endpoint titers were calculated as the reciprocal serum dilution giving signal 2 times background using a fitted curve (4-parameter log regression).

Confocal microscopy. LNs were harvested into O.C.T. Embedding Compound (Tissue-Tek, Sakura Finetek) 14 days after vaccination, snap-frozen, and cryosectioned into 7- $\mu \mathrm{m}$ slices (Leica). Dried sections were fixed in acetone for 10 minutes before blocking with 5\% (w/v) BSA and 2\% normal goat serum. Cell staining was 
performed using the following antibodies: B220 BV480 (RA3-6B2, BD), GL7 Alexa Fluor 488 (GL7, BioLegend), and CD35 BV421 (8C12, BD). Slides were mounted with ProLong Diamond Antifade Mountant (Life Technologies, Thermo Fisher Scientific). Images were recorded on a ZEISS Laser Scanning Confocal (LSM710) with 20×/0.8 NA air objective at 1 Airy unit, located at the Biological Optical Microscopy Platform at the University of Melbourne. Postprocessing of images was performed with FIJI (version 1.0).

Lightsheet microscopy. Methods are described in detail elsewhere (48). Fat-excised LNs were harvested into PLP buffer (1\% paraformaldehyde, $2 \mathrm{mg} / \mathrm{mL} \mathrm{NaIO}_{4}, 0.1 \mathrm{M}$ lysine, $0.05 \mathrm{M}$ phosphate buffer) and fixed 4-6 hours. LNs were transferred into blocking buffer containing 1\% normal goat serum, 1\% BSA, and $0.3 \%$ Triton X-100 in PBS and incubated overnight at room temperature (RT). LNs were then incubated 1:100 with IgD-Alexa Fluor 488 (11-26c.2a; BioLegend) and CD35 BV421 (8C12; BD) in blocking buffer for 3 days at RT. LNs were washed with PBS containing 0.2\% Triton X-100 and 0.5\% 1-thioglycerol for 24 hours. LNs were cleared for 24 hours at RT in 86\% Histodenz (MilliporeSigma), 0.5\% 1-thioglycerol, $0.1 \%$ Triton X-100, and $40 \% \mathrm{~N}$-methylacetamide. The clearing solution was replaced and LNs were cleared for a further 24 hours. The cleared LNs were embedded in $2 \%$ low-melting agarose and 1:10,000 Fluoresbrite YG Calibration beads ( $1 \mu \mathrm{m}$, Polysciences) inside sawn-off 1-mL syringes and submerged in clearing solution for 3 days before imaging. The refractive index of the clearing solution was matched throughout the protocol. Nodes were imaged on a ZEISS Z.1 lightsheet microscope with a $5 \times / 0.16$ air objective. The XY resolution was $1.31 \mu \mathrm{m}$, the $Z$ resolution was $5 \mu \mathrm{m}$, and the lightsheet thickness was $10.7 \mu \mathrm{m}$. Images were processed using Imaris (Bitplane).

Lymphocyte homogenates. Mouse LNs and spleens and macaque LNs were harvested into RF10 media (RPM I1640, 10\% FCS, 1× penicillin-streptomycin-glutamine; Life Technologies, Thermo Fisher Scientific). Single-cell suspensions were prepared by mechanical homogenization through a $70-\mu \mathrm{m}$ cell strainer. Mouse spleen cells and anticoagulant-collected mouse blood were then treated with Pharm Lyse (BD) to lyse red blood cells before subsequent use. Macaque LN homogenates were suspended in 90\% FCS with $10 \%$ DMSO and stored in liquid $\mathrm{N}_{2}$.

Flow cytometry detection of HA-specific murine B cells. Single-cell suspensions were stained with Aqua viability dye (Thermo Fisher Scientific) and Fc blocked with CD16/32 antibody (93; BioLegend). Cells were then surface stained with a PR8 HA probe and the following antibodies: B220 BUV737 (RA3-6B2; BD), IgD BUV395 (11-26c.2a; BD), CD45 Cy7-APC (30-F11; BD), GL7 Alexa Fluor 488 (GL7; BioLegend), CD38 Cy7/PE (90; BioLegend), streptavidin BV786 (BD), CD3 BV786 (145-2C11; BioLegend), and F4/80 BV786 (BM, BioLegend). Cells were washed twice and fixed with $1 \%$ formaldehyde (Polysciences). Flow cytometry data were acquired on a BD LSRFortessa using BD FACSDiva, analysis was performed using FlowJo v10, and statistical assessment was performed in GraphPad Prism.

Sequencing of antigen-specific antibodies and calculation of mutation rate. Murine immunoglobulin heavy chains were sequenced as previously described (49). Briefly, single-cell suspensions of lymphocytes were stained with Aqua viability dye (Thermo Fisher Scientific) and Fc blocked with CD16/32 antibody (93; BioLegend). Cells were then surface stained with a PR8 HA-PE and PR8 HA-BV421 probes and the following antibodies: IgD PerCP-Cy5.5 (11-26c2a; BD), F4/80 BV786 (BM8; BioLegend), CD3 BV786 (145-2C11; BioLegend), streptavidin- BV786 (BD), CD45 APC-Cy7 (30-F11; BD), B220 BV650 (RA3-6B2; BD), GL7 Alexa Fluor 488 (GL7; BioLegend), and CD38 PE-Cy7 (90; BioLegend). Single, class-switched, dual probe-positive GC or memory B cells were sorted into 96-well PCR plates using a BD FACSAria and stored at $-80^{\circ} \mathrm{C}$. mRNA was reverse-transcribed using random hexamers, and recombined heavy chain genes were amplified by nested PCR and sequenced (Macrogen). Mutations in the variable domains relative to C57BL/6 germline immunoglobulin genes were calculated using IMGT (www.imgt.org).

HAI assays. HAI assays were performed as previously described (50) using PR8 virus. Briefly, macaque immune sera were pretreated with receptor-destroying enzyme (RDE II, Denka Seiken), and HAI assays were performed using 4 hemagglutinating units per well and 1\% turkey red blood cells. HAI titers are expressed as the reciprocal of the highest dilution of serum at which hemagglutination was completely inhibited. Titers less than 20 were assigned as 10 .

Activation-induced marker assay to detect murine $T_{F H}$ responses. Based on published protocols used to identify antigen-specific $\mathrm{T}_{\mathrm{FH}}$ cells (18), freshly isolated LN cells were cultured in RF10 medium for 18 hours at $37^{\circ} \mathrm{C}$ with peptide pools ( $2 \mu \mathrm{g}$ /peptide/mL) spanning H. pylori ferritin (GenScript) or PR8 HA (BEI Resources). Peptide pools were generated from a peptide array of 17-mers overlapping by 11 amino acids, suspended in DMSO. Anti-CD154 BV650 (MR1, BioLegend) was added during stimulation. After stimulation, cells 
were recovered and stained with blue viability dye (Thermo Fisher Scientific), CD3 BV510 (145-2C11; BioLegend), CD25 BB515 (PD61; BD), PD-1 BV786 (29F.1A12; BioLegend), CXCR5 BV421 (L138D7; BioLegend), CD4 BUV737 (RM4-5; BD), OX-40 PE/Cy7 (OX-86; BioLegend), ICOS PerCP-eFluor 710 (15F9; Life Technologies, Thermo Fisher Scientific), B220 BV605 (RA3-6B2; BD), and F4/80 PE-CF594 (T45-2342; BD) before being washed, fixed, and acquired on a BD LSRFortessa using BD FACSDiva. Flow data were analyzed in FlowJo v10, and statistical assessment was performed in GraphPad Prism.

Identification of antigen-specific macaque $B$ cells and $T_{F H}$ cell activation. To identify antigen-specific B cells and activated $\mathrm{T}_{\mathrm{FH}}$ cells, defrosted LN cell suspensions were stained with PR8 HA-PE and PR8 HA-BV421 probes along with the following surface panel: CD14 BV510 (M5E2; BioLegend), CD16 BV510 (3G8; BioLegend), streptavidin BV510 (BD), Live/Dead Aqua (Thermo Fisher Scientific), CD4 BV605 (L200; BD), IgG BV786 (G18-145; BD), CXCR5 PE-Cy7 (MU5UBEE; Invitrogen, Thermo Fisher Scientific), CD20-Alexa Fluor 700 (2H7; BD), PD-1 APC Fire (EH12-2H7; BioLegend), IgD-Alexa Fluor 488 (polyclonal; Southern Biotech), IgM BUV395 (G20-127; BD), and CD3 BUV737 (SP34-2; BD). For Bc16-Alexa Fluor 647 (IG191E/A8; BioLegend) and Ki-67 BV650 (B56; BD) staining, cells were fixed, permeabilized, and stained using the BD Transcription Factor Buffer Kit according to the manufacturer's instructions. Samples were acquired on a BD LSRFortessa using BD FACSDiva. Flow data were analyzed in FlowJo v10, and statistical assessment was performed in GraphPad Prism.

Statistics. Data are presented as mean \pm SD unless otherwise stated. Statistical significance was assessed by Mann-Whitney $U$ test or Wilcoxon's matched-pairs signed-rank test (Figure 7, B-D) and performed using Prism version 7 (GraphPad). $P$ values less than 0.05 were considered statistically significant and marked with 1 asterisk. $P$ values less than 0.01 were marked with 2 asterisks.

Study approval. Mouse studies were approved by the University of Melbourne Animal Ethics Committee (number 1714104). Macaque studies (Macaca nemestrina) were approved by the Commonwealth Scientific and Industrial Research Organisation Animal Health Animal Ethics Committee (number 1833).

\section{Author contributions}

HGK, HXT, JAJ, SJK, and AKW designed the study. HGK, HXT, RE, WJ, and AKW performed experiments. VCW, BCD, and JRG facilitated lightsheet microscopy. YJ and FC facilitated TEM. MK provided ferritin expression constructs and facilitated protein purification methods. HGK, HXT, SJK, and AKW wrote the manuscript. All authors read and revised the manuscript.

\section{Acknowledgments}

The authors acknowledge the facilities and the scientific and technical assistance of the Biological Optical Microscopy Platform, the University of Melbourne. We also thank the expertise of Melbourne Cytometry Platform, specifically Vanta Jameson and Sarvy Taghavi. TEM analyses were conducted using the facilities at Bio21 Advanced Microscopy Facility, the University of Melbourne. HAI assay reagents and advice were provided by Paul Whitney, World Health Organization Collaborating Centre for Reference and Research on Influenza, Melbourne, and Mingyang Wang, Department of Microbiology and Immunology, The Peter Doherty Institute for Infection and Immunity, the University of Melbourne. The following reagent was obtained through BEI Resources, NIAID, NIH: Peptide Array, Influenza Virus A/ Puerto Rico/8/1934 (H1N1) Hemagglutinin Protein, NR-18973. This study was supported by the ARC Centre of Excellence in Convergent Bio-Nano Science and Technology Project CE140100036 (to SJK and FC), an NHMRC program grant APP1149990 (to SJK), and NHMRC project grants GNT1129099 and GNT1162760 (to AKW). JAJ, FC, SJK, and AKW are supported by NHMRC fellowships.

Address correspondence to: Stephen J. Kent or Adam K. Wheatley, Department of Microbiology and Immunology, Doherty Institute, 792 Elizabeth St., Melbourne, Victoria, Australia 3000. Phone 61.383449939; Email: skent@unimelb.edu.au (SJK). Phone: 61.383440764; Email: awheatley@unimelb.edu.au (AKW).

1. Gause KT, Wheatley AK, Cui J, Yan Y, Kent SJ, Caruso F. Immunological principles guiding the rational design of particles for vaccine delivery. ACS Nano. 2017;11(1):54-68.

2. Donaldson B, Lateef Z, Walker GF, Young SL, Ward VK. Virus-like particle vaccines: immunology and formulation for clinical translation. Expert Rev Vaccines. 2018;17(9):833-849.

3. Ingale J, et al. High-density array of well-ordered HIV-1 spikes on synthetic liposomal nanoparticles efficiently activate B cells. 
Cell Rep. 2016;15(9):1986-1999.

4. Thompson EA, et al. TLR-adjuvanted nanoparticle vaccines differentially influence the quality and longevity of responses to malaria antigen Pfs25. JCI Insight. 2018;3(10):120692.

5. Marcandalli J, et al. Induction of potent neutralizing antibody responses by a designed protein nanoparticle vaccine for respiratory syncytial virus. Cell. 2019;176(6):1420-1431.e17.

6. Yu F, et al. Nanoparticle-based adjuvant for enhanced protective efficacy of DNA vaccine Ag85A-ESAT-6-IL-21 against Mycobacterium tuberculosis infection. Nanomedicine. 2012;8(8):1337-1344.

7. Kanekiyo M, et al. Self-assembling influenza nanoparticle vaccines elicit broadly neutralizing H1N1 antibodies. Nature. 2013;499(7456):102-106.

8. Kelly HG, Kent SJ, Wheatley AK. Immunological basis for enhanced immunity of nanoparticle vaccines. Expert Rev Vaccines. 2019;18(3):269-280.

9. Link A, Zabel F, Schnetzler Y, Titz A, Brombacher F, Bachmann MF. Innate immunity mediates follicular transport of particulate but not soluble protein antigen. J Immunol. 2012;188(8):3724-3733.

10. Hu X, et al. Immune response of a novel ATR-AP205-001 conjugate anti-hypertensive vaccine. Sci Rep. 2017;7(1):12580.

11. Pardi N, et al. Nucleoside-modified mRNA vaccines induce potent $\mathrm{T}$ follicular helper and germinal center $\mathrm{B}$ cell responses. J Exp Med. 2018;215(6):1571-1588.

12. Tokatlian T, et al. Innate immune recognition of glycans targets HIV nanoparticle immunogens to germinal centers. Science. 2019;363(6427):649-654.

13. Whittle JR, et al. Flow cytometry reveals that $\mathrm{H} 5 \mathrm{~N} 1$ vaccination elicits cross-reactive stem-directed antibodies from multiple Ig heavy-chain lineages. $J$ Virol. 2014;88(8):4047-4057.

14. Tan HX, et al. Subdominance and poor intrinsic immunogenicity limit humoral immunity targeting influenza HA stem. $J C l i n$ Invest. 2019;129(2):850-862.

15. Mesin L, Ersching J, Victora GD. Germinal center B cell dynamics. Immunity. 2016;45(3):471-482.

16. Song W, Craft J. T follicular helper cell heterogeneity: time, space, and function. Immunol Rev. 2019;288(1):85-96.

17. Mitchison NA. T-cell-B-cell cooperation. Nat Rev Immunol. 2004;4(4):308-312.

18. Jiang W, et al. Identification of murine antigen-specific $\mathrm{T}$ follicular helper cells using an activation-induced marker assay. J Immunol Methods. 2019;467:48-57.

19. Corbett KS, et al. Design of nanoparticulate group 2 influenza virus hemagglutinin stem antigens that activate unmutated ancestor B cell receptors of broadly neutralizing antibody lineages. mBio. 2019;10(1):e02810-18.

20. Villar RF, et al. Reconstituted B cell receptor signaling reveals carbohydrate-dependent mode of activation. Sci Rep. 2016;6:36298.

21. Andrews SF, et al. Immune history profoundly affects broadly protective B cell responses to influenza. Sci Transl Med. 2015;7(316):316ra192

22. Moon JJ, Suh H, Li AV, Ockenhouse CF, Yadava A, Irvine DJ. Enhancing humoral responses to a malaria antigen with nanoparticle vaccines that expand Tfh cells and promote germinal center induction. Proc Natl Acad Sci U S A. 2012;109(4):1080-1085.

23. Phan TG, Grigorova I, Okada T, Cyster JG. Subcapsular encounter and complement-dependent transport of immune complexes by lymph node B cells. Nat Immunol. 2007;8(9):992-1000.

24. Tavano R, et al. C1q-mediated complement activation and C3 opsonization trigger recognition of stealth poly(2-methyl-2-oxazoline)-coated silica nanoparticles by human phagocytes. ACS Nano. 2018;12(6):5834-5847.

25. Chen F, et al. Complement proteins bind to nanoparticle protein corona and undergo dynamic exchange in vivo. Nat Nanotechnol. 2017;12(4):387-393

26. Liao W, Hua Z, Liu C, Lin L, Chen R, Hou B. Characterization of T-dependent and T-independent B cell responses to a viruslike particle. J Immunol. 2017;198(10):3846-3856.

27. Vinuesa CG, Linterman MA, Yu D, MacLennan IC. Follicular helper T cells. Annu Rev Immunol. 2016;34:335-368

28. Bachmann MF, Jennings GT. Vaccine delivery: a matter of size, geometry, kinetics and molecular patterns. Nat Rev Immunol. 2010;10(11):787-796.

29. Yassine HM, et al. Hemagglutinin-stem nanoparticles generate heterosubtypic influenza protection. Nat Med. 2015;21(9):1065-1070.

30. Brouwer PJM, et al. Enhancing and shaping the immunogenicity of native-like HIV-1 envelope trimers with a two-component protein nanoparticle. Nat Commun. 2019;10(1):4272.

31. Brinkkemper M, Sliepen K. Nanoparticle vaccines for inducing HIV-1 neutralizing antibodies. Vaccines (Basel). $2019 ; 7(3)$ :E76.

32. Mueller J, Matloubian M, Zikherman J. Cutting edge: an in vivo reporter reveals active B cell receptor signaling in the germinal center. J Immunol. 2015;194(7):2993-2997.

33. Sliepen K, et al. Presenting native-like HIV-1 envelope trimers on ferritin nanoparticles improves their immunogenicity. Retrovirology. 2015;12:82.

34. Sliepen K, et al. Structure and immunogenicity of a stabilized HIV-1 envelope trimer based on a group-M consensus sequence. Nat Commun. 2019;10(1):2355.

35. $\mathrm{Bu} \mathrm{W}$, et al. Immunization with components of the viral fusion apparatus elicits antibodies that neutralize Epstein-Barr virus in B cells and epithelial cells. Immunity. 2019;50(5):1305-1316.e6.

36. Kanekiyo M, et al. Rational design of an Epstein-Barr virus vaccine targeting the receptor-binding site. Cell. 2015;162(5):1090-1100.

37. Luckey D, Weaver EA, Osborne DG, Billadeau DD, Taneja V. Immunity to influenza is dependent on MHC II polymorphism: study with 2 HLA transgenic strains. Sci Rep. 2019;9(1):19061.

38. Boldt AB, et al. Phylogenetic nomenclature and evolution of mannose-binding lectin (MBL2) haplotypes. BMC Genet. 2010;11:38

39. Minchinton RM, Dean MM, Clark TR, Heatley S, Mullighan CG. Analysis of the relationship between mannose-binding lectin (MBL) genotype, MBL levels and function in an Australian blood donor population. Scand J Immunol. 2002;56(6):630-641.

40. Chang WC, et al. Lack of the pattern recognition molecule mannose-binding lectin increases susceptibility to influenza A virus infection. BMC Immunol. 2010;11:64.

41. Shi L, et al. Mannose-binding lectin-deficient mice are susceptible to infection with Staphylococcus aureus. J Exp Med. 
2004;199(10):1379-1390.

42. Carter T, et al. Mannose-binding lectin A-deficient mice have abrogated antigen-specific IgM responses and increased susceptibility to a nematode infection. J Immunol. 2007;178(8):5116-5123.

43. Dahl M, Tybjaerg-Hansen A, Schnohr P, Nordestgaard BG. A population-based study of morbidity and mortality in mannose-binding lectin deficiency. J Exp Med. 2004;199(10):1391-1399.

44. Verga Falzacappa MV, Segat L, Puppini B, Amoroso A, Crovella S. Evolution of the mannose-binding lectin gene in primates Genes Immun. 2004;5(8):653-661.

45. Althaus CL. Of mice, macaques and men: scaling of virus dynamics and immune responses. Front Microbiol. 2015;6:355.

46. Cirelli KM, et al. Slow delivery immunization enhances HIV neutralizing antibody and germinal center responses via modulation of immunodominance. Cell. 2019;177(5):1153-1171.e28.

47. Havenar-Daughton C, et al. Rapid germinal center and antibody responses in non-human primates after a single nanoparticle vaccine immunization. Cell Rep. 2019;29(7):1756-1766.e8.

48. Li W, Germain RN, Gerner MY. Multiplex, quantitative cellular analysis in large tissue volumes with clearing-enhanced 3D microscopy (C 3D). Proc Natl Acad Sci U S A. 2017;114(35):E7321-E7330.

49. von Boehmer L, et al. Sequencing and cloning of antigen-specific antibodies from mouse memory B cells. Nat Protoc. 2016;11(10):1908-1923.

50. Kristensen $\mathrm{AB}$, et al. Antibody responses with Fc-mediated functions after vaccination of $\mathrm{HIV}$-infected subjects with trivalent influenza vaccine. J Virol. 2016;90(12):5724-5734. 